\title{
Voluntariado: uma Avaliação da Motivação entre Acadêmicos de Medicina e da Experiência no Projeto "Cuidando da Sua Saúde em Ponto dos Volantes, Jequitinhonha, MG"
}

\author{
Volunteering: an Assessment of the \\ Motivation among Medical Students and \\ the Experience in the Project "Caring \\ for Your Health in Ponto dos Volantes, \\ Jequitinhonha, $M G$ "
}

\author{
Fernanda Mendes Amorim ${ }^{l}$ \\ Ana Paula Bernardes Real \\ Gustavo Antunes Rodrigues Duartel \\ Juliana Toledo Mesquital \\ Bruno Cézar Lage Cotal \\ Luís Felipe José Ravic de Miranda
}

\section{PALAVRAS-CHAVE}

- Voluntariado.

- Atendimento Médico.

- Estudantes de Medicina.

- Motivação.
Objetivo: Trata-se de um estudo descritivo, dividido em duas abordagens distintas, a respeito do trabalho voluntário entre estudantes de Medicina. Este artigo buscou compreender o nivel de engajamento desses estudantes em projetos voluntários e as repercussões geradas com a atuação em um projeto de voluntariado da Universidade Federal de Minas Gerais (UFMG) em um grupo de alunos. Método: O estudo foi realizado em duas partes. A primeira parte trata-se de um estudo transversal de análise quantitativa que, por meio de um questionário online na plataforma Google Forms, entrevistou 135 acadêmicos de Medicina da Universidade Federal de Minas Gerais (UFMG) do primeiro ao décimo segundo período. Os participantes foram questionados sobre já terem ou não se engajado em serviços voluntários e os motivos deste engajamento. A segunda parte do artigo versa sobre um estudo transversal de análise qualitativa que avaliou o impacto do projeto voluntário "Cuidando da sua saúde em Ponto dos Volantes", promovido pela Faculdade de Medicina da UFMG, na vida dos participantes. O projeto ocorreu em julho de 2017 no Vale do Jequitinhonha (MG), com 19 integrantes, todos alunos da graduação de Medicina. Foram avaliadas, por meio da plataforma Google Forms, as opiniões de 15 dos 19 estudantes participantes do projeto. Resultados: Os resultados do primeiro estudo mostraram que 108 (80\%) dos participantes já haviam feito algum trabalho voluntário na vida e 97,3\% deles gostariam de participar de outra atividade semelhante. Quando questionados sobre os benefícios que o voluntariado proporcionou, o crescimento pessoal foi a alternativa mais selecionada, com 94 (25,06\%) votos dentre o total de 375 votos; o incremento do curriculum vitae não ficou entre os mais votados, tendo obtido somente 23 $(6,13 \%)$ votos. Entre as pessoas sem interesse em se dedicar ao voluntariado, a falta de tempo foi o motivo mais escolhido. Além disso, $110(81,48 \%)$ dos participantes da pesquisa afirmam ter desejo de trabalhar como voluntários após a graduação. No segundo estudo, todos os 15 alunos (100\%) que responderam ao questionário mostraram interesse em realizar novo trabalho voluntário. Quando indagados sobre o impacto do trabalho em suas vidas, apenas aspectos positivos foram levantados, como valorização da própria vida e da do próximo. Quanto ao motivo de se engajar no projeto, a grande maioria mencionou a possibilidade de conhecer realidades distintas da cotidiana e dos serviços da faculdade. Conclusão: Juntos, ambos os estudos mostram que o trabalho voluntário tem alcançado notoriedade na vida dos estudantes de Medicina da UFMG e que o não engajamento em tais projetos ocorre mais por falta de conhecimento e divulgação destes do que pela falta de interesse dos alunos. Nosso estudo concluiu ser necessário maior investimento na criação e divulgação de projetos voluntários acadêmicos e acredita que essa realidade deva ser expandida não só nas universidades ao longo do País, mas em escolas e em comunidades como um todo. 


\section{KEY-WORDS}

- Volunteering.

- Medical Care.

- Medical Students.

- Motivation.

Recebido em: 2/7/19

Aceito em: 22/7/19
ABSTRACT

Objective: This is a descriptive study divided into two distinct approaches to voluntary work among medical students. This article sought to understand the level of engagement of these students in voluntary projects and the repercussions generated by working in a volunteer project of the Federal University of Minas Gerais (UFMG) in a group of students. Methods: The study was carried out in two parts. The first part is a cross-sectional study involving quantitative analysis, through which, with the use of an online questionnaire in Google Forms, 135 first- to sixth-year medical students from the Federal University of Minas Gerais (UFMG) were interviewed. Participants were asked whether or not they had engaged in voluntary services and the reasons for doing so. The second part of the article is a cross-sectional, qualitative analysis that evaluated the impact of the voluntary project "Caring for your health in Ponto dos Volantes", promoted by the UFMG Medical School on the life of the participants. The project took place in July 2017 in Vale do Jequitinhonha, Minas Gerais, with 19 members, all of them undergraduate medical students. The opinions of 15 of the 19 students participating in the project were evaluated through the Google Forms platform. Results: The results of the first study showed that 108 (80\%) of the participants had previously done some voluntary work and $97.3 \%$ of them would like to participate in another similar activity. When questioned about the benefits that volunteering provided, personal growth was the most common answer, with $94(25.06 \%)$ votes out of 375 votes; enhancing one's curriculum vitae was not among the most voted, obtaining only $23(6.13 \%)$ votes. Among those with no interest in volunteering, lack of time was the most common reason. In addition, 110 (81.48\%) of the study participants stated that they would like to volunteer after graduation. In the second study, all 15 students (100\%) who answered the questionnaire showed interest in carrying out new voluntary work. When asked about the impact of volunteering on their lives, only positive aspects were raised, such as valuing one's life and that of others. As for the reason for engaging in the project, the vast majority mentioned the possibility of learning about different realities from daily life and college services. Conclusion: Together, the two studies show that voluntary work has become a well-known feature in the life of UFMG medical students and that non-engagement in such projects is more due to lack of knowledge and dissemination than to lack of interest. Our study concluded that greater investment in the creation and dissemination of academic voluntary projects is needed and that this reality should be expanded not only within universities across the country but in schools and communities as a whole.

\section{INTRODUÇÃO}

O trabalho voluntário é considerado uma forma de trabalho não compulsório e assalariado por meio de uma organização ou diretamente para outras pessoas com quem não se tenha grau de parentesco ${ }^{1}$. Os benefícios que esta atividade produz nos indivíduos são sugeridos por estudos e estão relacionados a bem-estar social, emocional e psicológico, e a efeitos positivos sobre a saúde ${ }^{2,3}$. Apesar disso, no Brasil, em 2016, apenas $3,9 \%$ da população acima de 14 anos realizaram algum tipo de trabalho voluntário ; a título de comparação, em 2015, nos Estados Unidos, 24,9\% da população estavam envolvidos com esse tipo de atividade ${ }^{5}$.

Os motivos que levam as pessoas a realizarem um trabalho voluntário são diversos. Em 1998, Clary et al. ${ }^{6}$ organizaram em seis grupos as principais motivações para os indivíduos se voluntariarem. Estas motivações foram: valores, como uma forma de expressar preocupações humanitárias e altruísticas; compreensão, o voluntariado proporciona o aprendizado de novas experiências e o uso de conhecimento e habilidades; social, o trabalho voluntário é uma maneira de estar com amigos; carreira, uma forma de usar os benefícios do trabalho voluntário, como o uso de habilidades; protetor, como uma forma de reduzir a culpa por ser mais afortunado na vida, por exemplo; e aprimoramento, o voluntariado permite satisfação com o crescimento pessoal e autoestima.

A medicina é uma carreira que exige um papel humanitário dos profissionais para seu bom exercício, uma vez que envolve o contato direto com as pessoas em um papel de curar, remediar, prevenir e aliviar um sofrimento. Ser solidário é uma característica necessária à profissão ${ }^{7}$. Considerando a atuação de um médico, é plausível pensar que as pessoas que estudam Medicina possuem grande motivação para o trabalho voluntário e devem se envolver com esta atividade.

Segundo o Instituto Brasileiro de Geografia e Estatística

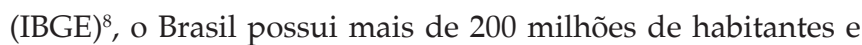
contava, em janeiro de 2018, com 452.801 médicos, numa re- 
lação de aproximadamente 2,18 médicos por mil habitantes e distribuídos de maneira desigual no território brasileiro. A região com maior densidade médica é o Sudeste, com 2,81 médicos por mil habitantes, enquanto a Região Norte apresenta uma relação de 1,16, e a Região Nordeste, de 1,41. A comparação entre a quantidade de médicos nas capitais e no interior dos estados evidencia ainda mais essa desigualdade, uma vez que as capitais concentram cerca de um quarto da população brasileira e mais da metade dos médicos. Há regiões do interior do Nordeste que apresentam uma relação menor que um médico para cada mil habitantes ${ }^{9}$.

Com a expectativa de se formarem mais de 20 mil médicos no Brasil a partir de 2018, a identificação e o combate da desigualdade de médicos nas regiões do País se tornam essenciais. Esta situação demonstra a importância de uma formação médica mais humana, sensível e consciente das reais condições sociais e econômicas da população brasileira. $\mathrm{O}$ fomento e o exercício do voluntariado no período da graduação se apresentam como medidas para alcançar este objetivo.

Com base na pequena expressão do voluntariado na população brasileira e na importância que esta atividade tem a desempenhar entre os futuros médicos, este artigo apresenta um estudo descritivo, promocional do voluntariado, na cidade de Ponto dos Volantes, município situado na região do Vale do Jequitinhonha, Minas Gerais, com população estimada de 12.138 habitantes. e IDH menor que a média nacional, 0.595 e 0.744 respectivamente. A média salarial não ultrapassa 1.5 salários mínimos, e o município apresenta $0 \%$ de domicílios urbanos em vias públicas com urbanização adequada (presença de bueiro, calçada, pavimentação e meio-fio) ${ }^{10}$.

O projeto foi custeado pela prefeitura da cidade, a qual arcou com as despesas de transporte, alimentação e acomodação dos alunos, sendo o seguro viagem fornecido pela UFMG. Apesar de mais de dez laboratórios farmacêuticos terem sido contatados, nenhum deles se dispôs a patrocinar o projeto.

\section{OBJETIVOS}

\section{Principais}

O projeto teve o objetivo de proporcionar aos estudantes maior conhecimento sobre a realidade da saúde de uma população que não vive próxima a grandes centros urbanos e estimular uma visão mais humanitária e altruística. Avaliou também as motivações da participação no voluntariado e o impacto que o envolvimento em um projeto social ("Cuidando da sua saúde em Ponto dos Volantes") pode ter nos estudantes de Medicina.

\section{Secundários}

Os objetivos secundários consistiram em oferecer atendimento médico à população local; fornecer informações básicas a respeito das doenças mais prevalentes na área clínica; avaliar as taxas de adesão terapêutica e sua correlação com o conhecimento das comorbidades.

\section{MÉTODO DO ESTUDO 1}

Foi realizado um estudo transversal com base numa entrevista, por meio da plataforma online Google Forms, com 135 estudantes de Medicina da Universidade Federal de Minas Gerais (UFMG), do primeiro ao décimo segundo período do curso, no período de 18 de setembro de 2017 a 29 de novembro de 2017 (Tabela 1). Os questionários foram inspirados nas seis funções do voluntariado ${ }^{6}$ e foram divulgados por meio das redes sociais. Os participantes foram questionados sobre já terem feito algum trabalho voluntário e o interesse que possuem em se engajar. Foram dadas alternativas que justificassem o interesse ou não em voluntariado e um espaço opcional para comentários.

\begin{tabular}{|ll|}
\hline & \multicolumn{1}{c}{ TABELA 1.} \\
\hline Período & Número de alunos por período do curso \\
\hline 1 & $4(2,96)$ \\
\hline 2 & $8(5,92)$ \\
\hline 3 & $5(3,70)$ \\
\hline 4 & $7(5,18)$ \\
\hline 5 & $7(5,18)$ \\
\hline 6 & $13(9,62)$ \\
\hline 7 & $16(11,85)$ \\
\hline 8 & $22(16,29)$ \\
\hline 9 & $31(22,96)$ \\
\hline 10 & $5(3,70)$ \\
\hline 11 & $15(11,11)$ \\
\hline 12 & $2(1,48)$ \\
\hline
\end{tabular}

Após a entrevista, uma análise quantitativa foi realizada para avaliar as respostas dos estudantes às seguintes perguntas:

1. Você sabe o que é voluntariado?

2. Você já fez algum trabalho voluntário?

3. Se já fez algum trabalho voluntário, responda:

a. Foi antes da Faculdade de Medicina

b. Foi durante a Faculdade de Medicina

c. Antes e durante a faculdade

4. O trabalho foi na área da saúde?

5. Se já fez trabalho voluntário, o que trouxe de benefício pessoal?

a. Crescimento pessoal 
b. Habilidades com pessoas

c. Certificado e incremento no currículo

d. Mais valorização da vida

e. Menos preconceito

f. Menos valor a bens materiais

g. Maior conhecimento de diferentes realidades

h. Novos vínculos e amizades

6. Faria algum trabalho voluntário novamente? Se já fez alguma vez na vida.

7. Conhece algum projeto de voluntariado na UFMG?

8. Se você TEM interesse em participar de algum trabalho voluntário e acha importante, marque as razões:
a. Crescimento pessoal
b. Conhecer outras realidades
c. Vontade de ajudar as pessoas
d. Currículo
e. Recomendação de alguém
f. Tentar fazer novos amigos e conhecer pessoas
g. Buscar um mundo com mais igualdade
h. Influência religiosa
i. Reduzir preconceitos
j. Dar bons exemplos na sociedade

9. Se você NÃO TEM interesse em participar de algum trabalho voluntário, marque as razões:
a. Falta de tempo
b. Falta de motivação
c. Nunca tentou
d. Falta de conhecimento de projetos de voluntariado
e. Não há projetos próximos de casa
f. Falta de companhia para entrar em um projeto
g. Experiência negativa anterior

10. Tem interesse em fazer trabalho voluntário depois de se formar?

11. Tem interesse em fazer trabalho voluntário na área médica?

12. Comentários

\section{RESULTADOS DO ESTUDO 1}

Dos 135 alunos que responderam ao questionário online, 108 $(80 \%)$ afirmaram que já fizeram algum trabalho voluntário na vida, e este trabalho ocorreu antes e durante a Faculdade de Medicina para 45 (41,7\%) pessoas. Para 37 (34,3\%) participantes, o trabalho foi antes da faculdade, e para $26(24,1 \%)$, apenas durante o curso. Do total, 46 (40,4\%) alunos responderam que este trabalho foi na área da saúde e 97,3\% responderam que fariam um trabalho voluntário novamente.

Os 108 participantes que afirmaram ter participado de algum trabalho voluntário foram questionados sobre o benefício pessoal que o voluntariado trouxe para suas vidas (Tabela 2).
Das 8 opções dadas como benefício, os estudantes marcaram as que consideraram mais significativas. $\mathrm{O}$ crescimento pessoal foi a opção mais selecionada, com 94 votos. Possibilidade de maior conhecimento de diferentes realidades, desenvolvimento de habilidades com pessoas e maior valorização da vida foram outras opções mais selecionadas, com 66, 55 e 49 votos respectivamente. Vinte e três pessoas indicaram o ganho de um certificado e o incremento do curriculum vitae como um benefício significativo do trabalho voluntário.

\begin{tabular}{|c|c|c|c|}
\hline \multicolumn{4}{|c|}{$\begin{array}{l}\text { TABELA } 2 . \\
\text { O que o trabalho voluntário trouxe de benefícios pessoal }\end{array}$} \\
\hline Respostas & Votos & $\begin{array}{l}\text { Votos por } \\
\text { número } \\
\text { total de } \\
\text { votos } \\
(375)\end{array}$ & $\begin{array}{c}\text { Votos por número } \\
\text { de participantes } \\
\text { que já fizeram } \\
\text { trabalho } \\
\text { voluntário (108) }\end{array}$ \\
\hline Crescimento pessoal & 94 & $25,06 \%$ & $87,0 \%$ \\
\hline Habilidades com pessoas & 55 & $14,66 \%$ & $50,9 \%$ \\
\hline $\begin{array}{l}\text { Certificado e incremento } \\
\text { no currículo }\end{array}$ & 23 & $6,13 \%$ & $21,3 \%$ \\
\hline Mais valorização à vida & 49 & $13,06 \%$ & $45,4 \%$ \\
\hline Menos preconceito & 31 & $8,26 \%$ & $28,7 \%$ \\
\hline $\begin{array}{l}\text { Dar menos valor a bens } \\
\text { materiais }\end{array}$ & 32 & $8,53 \%$ & $29,6 \%$ \\
\hline $\begin{array}{l}\text { Maior conhecimento de } \\
\text { diferentes realidades }\end{array}$ & 66 & $17,60 \%$ & $61,1 \%$ \\
\hline $\begin{array}{l}\text { Novos vínculos e } \\
\text { amizades }\end{array}$ & 25 & $6,66 \%$ & $23,1 \%$ \\
\hline
\end{tabular}

Quando os estudantes foram questionados sobre o interesse em atuar no voluntariado após se formarem, 110 (81,48\%) afirmaram que têm interesse e 111 (82,22\%) afirmaram desejar atuar na área médica; 22 (16,29\%) responderam que ainda não sabem e $3(2,22 \%)$ responderam que não possuem interesse.

Os estudantes que têm interesse em participar de algum projeto de voluntariado e consideram o trabalho importante indicaram as principais razões, entre dez fornecidas (Tabela 3). Dentre todos, 131 alunos responderam a este questionamento. Vontade de ajudar as pessoas, crescimento pessoal e conhecimento de outras realidades foram as opções mais consideradas. Recomendação de alguém e influência religiosa foram as opções menos selecionadas.

Quando os participantes foram questionados sobre a ausência de interesse em participar de um trabalho voluntário, 18 responderam às principais razões dentre as seis fornecidas (Tabela 4). Falta de tempo foi o principal motivo, com 16 votos, seguido da falta de conhecimento de projetos de voluntariado e da falta de motivação, com 9 e 6 votos, respectivamente.

Dentre os entrevistados, 59,3\% afirmaram não conhecer nenhum projeto de voluntariado fomentado pela universidade. 
TABEla 3.

As razões para participar em trabalhos voluntários pelos estudantes com interesse no voluntariado.

\begin{tabular}{lccc}
\hline Respostas & Votos & $\begin{array}{c}\text { Votos por } \\
\text { número } \\
\text { total de } \\
\text { votos }\end{array}$ & $\begin{array}{c}\text { Votos por número de } \\
\text { estudantes que tem } \\
\text { interesse em trabalho } \\
\text { voluntário (131) }\end{array}$ \\
\hline $\begin{array}{l}\text { Crescimento pessoal } \\
\text { Conhecer outras }\end{array}$ & 92 & $20,86 \%$ & $70,2 \%$ \\
realidades & 89 & $20,18 \%$ & $67,9 \%$ \\
\hline $\begin{array}{l}\text { Vontade de ajudar ao } \\
\text { próximo }\end{array}$ & 99 & $22,44 \%$ & $75,6 \%$ \\
\hline $\begin{array}{l}\text { Currículo } \\
\text { Recomendação de } \\
\text { alguém }\end{array}$ & 23 & $5,21 \%$ & $17,6 \%$ \\
\hline $\begin{array}{l}\text { Tentar fazer novas } \\
\text { amizades e conhecer } \\
\text { pessoas diferentes }\end{array}$ & 14 & $3,17 \%$ & $4,6 \%$ \\
\hline $\begin{array}{l}\text { Buscar um mundo com } \\
\text { mais igualdade }\end{array}$ & 44 & $9,97 \%$ & $10,7 \%$ \\
\hline $\begin{array}{l}\text { Influência religiosa } \\
\text { Reduzir preconceitos }\end{array}$ & 12 & $2,72 \%$ & $33 \%$ \\
\hline $\begin{array}{l}\text { Dar bons exemplos à } \\
\text { sociedade }\end{array}$ & 29 & $6,57 \%$ & $22,1 \%$ \\
\hline & & $7,48 \%$ & $9,2 \%$ \\
\hline
\end{tabular}

\begin{tabular}{|c|c|}
\hline \multicolumn{2}{|c|}{$\begin{array}{l}\text { TABELA } 4 . \\
\text { As razões para não participar em trabalhos } \\
\text { voluntários pelos estudantes que não } \\
\text { tinham interessa no voluntariado }\end{array}$} \\
\hline Respostas & Votos \\
\hline Falta de tempo & $16(88,9 \%)$ \\
\hline Falta de motivação & $6(33,3 \%)$ \\
\hline Nunca tentou & $3(16,7 \%)$ \\
\hline Falta de conhecimento de projetos voluntariados & $9(50,0 \%)$ \\
\hline Não há projetos próximos de casa & $2(11,1 \%)$ \\
\hline Falta de companhia para entrar em um projeto & $3(16,7 \%)$ \\
\hline Experiência negativa anterior & $0(0 \%)$ \\
\hline
\end{tabular}

\section{MÉTODO DO ESTUDO 2}

A segunda parte do estudo avaliou a opinião de 15 estudantes de Medicina que participaram de um trabalho voluntário em julho de 2017, intitulado "Cuidando da sua saúde em Ponto dos Volantes". Os autores deste artigo, por terem participado do projeto, não responderam ao questionário, não havendo, assim, viés na análise.

Um estudo transversal foi realizado tendo por base entrevistas anônimas, por meio da plataforma online Google Forms, com 15 estudantes de Medicina do quinto ao décimo segundo período do curso que participaram do projeto "Cuidando da sua saúde em Ponto dos Volantes". O projeto foi uma ação de voluntariado, desenvolvida por um professor do Departamento de Clínica Médica (CLM) e aprovado pela câmara departamental da CLM da Faculdade de Medicina da UFMG.

No período de 24 a 28 de julho de 2017, 19 acadêmicos de Medicina que cursavam do quinto ao décimo primeiro período, supervisionados por dois professores coordenadores, estiveram no município de Ponto dos Volantes, Vale do Jequitinhonha, Minas Gerais. Os alunos foram divididos em quatro pequenos grupos, e cada um deles ficou responsável por uma atividade específica desenvolvida com a comunidade local.

Dois desses grupos ficaram responsáveis pelo atendimento clínico aos pacientes, tanto na área urbana, quanto na zona rural do município. No total, foram realizados 84 atendimentos, voltados para adultos e idosos.

Outro grupo realizou atividades no ambulatório de pequenas cirurgias, onde foram feitos 33 procedimentos, incluindo cantoplastia, exérese de fibromas moles, lipomas, cistos sebáceos, nevos melanocíticos, verruga vulgar e biópsias de pele suspeitas de carcinoma.

O quarto grupo de alunos se responsabilizou pelas aulas teóricas, nas quais foram abordados temas de grande relevância para a população assistida: hipertensão arterial sistêmica, diabetes mellitus, tabagismo e depressão.

Os alunos conduziram as atividades, revezavam-se diariamente entre os grupos descritos acima e foram supervisionados pelos médicos professores da equipe, contando sempre com o auxílio dos profissionais de saúde locais.

Após a realização do projeto e das entrevistas, fez-se uma análise qualitativa das respostas dos estudantes que participaram. Os alunos responderam às seguintes perguntas:

1. Qual é o conceito de voluntariado para você?

2. Mudou o conceito que tinha após a experiência em Ponto dos Volantes?

3. Você já fez trabalho voluntário? Antes ou durante a faculdade?

4. Por que você quis entrar para o projeto de Ponto dos Volantes?

5. Você já conhecia a realidade de parte da população brasileira com relação à saúde?

6. Qual impacto o trabalho produziu na sua vida? Mudança de mentalidade, estilo de vida, hábitos?

7. O que você busca com o trabalho voluntário?

8. O que a população local the ensinou?

9. Faria novamente um trabalho voluntário como este?

10. Algo a acrescentar? (Alguma experiência negativa, expectativas, comentários...) 


\section{RESULTADOS DO ESTUDO 2}

Todos os 15 alunos que responderam ao questionário mostraram interesse em realizar novo trabalho voluntário. Onze deles já haviam realizado trabalho voluntário, ou antes ou durante o período da faculdade.

A primeira pergunta foi a respeito do conhecimento dos participantes sobre o conceito de voluntariado. Os estudantes entendem que o trabalho voluntário é não remunerado e se volta para uma pessoa ou grupo de pessoas e interpretam a atividade como um ato de solidariedade. Denotam essa atitude algumas respostas como: (a) "doar-se para o outro", (b) "ajudar sem ter um retorno financeiro em troca", (c) "é ação de solidariedade. Materialização de amor ao próximo. Não é apenas ajudar, é deixar algo concreto no coração das pessoas que necessitam".

A segunda pergunta indagava se o conceito de voluntariado reforçava ou mudava a ideia prévia desse assunto. Da totalidade dos alunos, nove afirmaram que reforçava e sete perceberam que era algo mais abrangente do que tinham em mente. Além disso, quatro deles nunca haviam realizado alguma atividade de voluntariado.

Com relação às razões que motivaram a aceitação em participar do projeto, a grande maioria levantou a questão de conhecer uma nova realidade mais de perto e sair da zona de conforto, visto que mais da metade dos acadêmicos alegou nunca ter tido contato com grupos populacionais tão distintos dos assistidos nas unidades básicas de saúde de Belo Horizonte. Os participantes apontaram razões semelhantes, como: (a) "ver uma realidade totalmente diferente da que estou acostumado e tentar fazer a diferença na vida de pessoas que têm tão pouco", (b) "conhecer outra realidade diferente da minha, para viver a experiência de um local mais pobre e pouco acessível", (c) "trabalho médico voluntário sempre foi algo que admirei e que tinha vontade de fazer, porém não tive muitas oportunidades", (d) "oportunidade de conhecer outras realidades e ajudar", (e) "seria minha chance para fazer de fato um trabalho voluntário em uma área em que sei que posso dar um pouco de ajuda", (f) "a ideia do projeto sempre me pareceu muito interessante: conhecer uma realidade completamente diferente daquela com a qual estamos acostumados dentro desse ambiente central de Belo Horizonte, além de oferecer uma atenção de qualidade a uma população de uma região carente do estado", (g) "oportunidade de conhecer a realidade do Vale do Jequitinhonha e contribuir com a saúde da população local, além de poder aplicar meus conhecimentos em benefício das pessoas. Sempre tive vontade de participar de um projeto como esse e quando surgiu a oportunidade não poderia deixar de ir".
Quando os estudantes foram questionados sobre o conhecimento que tinham das reais condições sociais e econômicas de parte da população brasileira, muitos expressaram apenas uma consciência parcial. Algumas das respostas foram: (a) "achava que conhecia, mas vi algo totalmente diferente e aquém do que eu imaginava", (b) "somente por relatos de colegas ou pela mídia, não tinha vivenciado essa realidade ainda", (c) "conhecia, mas não tinha ideia da proporção dessa realidade, já que não havia tido um contato tão próximo com ela", (d) "apenas através da mídia e do contato com pacientes que vêm do interior para atendimento médico", (e) "conheci apenas durante a faculdade", (f) "a realidade vista em Ponto dos Volantes foi além da minha expectativa no que diz respeito à pobreza social", (g) "já estive em outros locais com realidades semelhantes àquelas ou em alguns casos até piores".

A análise qualitativa a respeito do impacto do trabalho na vida do estudante resultou em respostas positivas, como (a) "acho que reclamo menos das coisas; um hábito que adquiri depois da experiência foi sempre lembrar dos momentos que vivi lá e compará-los com minha atual situação", (b) "percebi em mim um egoísmo que nem eu sabia que existia. $\mathrm{O}$ voluntariado quebrou isso", (c) "o trabalho me fez perceber o quanto ainda tenho para aprender", (d) "com o trabalho em Ponto dos Volantes aprendi que é mais fácil do que parece ajudar as pessoas". Os participantes demonstraram o que buscam no trabalho voluntário: (a) "ampliar meus conhecimentos sobre o outro, sobre o que me cerca e não vejo, para ser melhor como profissional de saúde e atender com mais carinho e respeito, (b) "ajudar as pessoas a terem acesso à saúde com mais dignidade" e (c) "ofertar minhas habilidades, tempo e conhecimento em benefício de pessoas que necessitam de melhor atendimento médico e maior acesso à saúde".

Também é relevante avaliar o que se obteve de aprendizado com a população local: "humildade", "respeito" e "possibilidade de viver bem com muito menos" foram os mais citados no questionário.

\section{DISCUSSÃO}

Os dois estudos deste artigo demonstraram qual é a motivação de estudantes de Medicina em relação ao voluntariado e como aqueles que já participaram de um projeto avaliam esta atividade e seu impacto em suas vidas. O Estudo 1 apresentou que $81,5 \%$ dos 135 entrevistados têm interesse em atuar no voluntariado após a graduação, e apenas 40,7\% conhecem algum projeto na área desenvolvido por sua universidade. No Estudo 2, os estudantes se expressaram de forma livre e anônima. Todos os alunos participantes do projeto afirmaram que voltariam a fazer trabalho voluntário. Dessa forma, demonstra-se 
como a experiência e a oportunidade de atuar no voluntariado estimulam os alunos a se dedicarem a este tipo de atividade.

Os participantes do projeto reconheceram os benefícios que o trabalho voluntário pode ter em suas vidas e nas de outras pessoas. No Estudo 1, crescimento pessoal e aquisição de conhecimento sobre a realidade de grande parte da população brasileira foram alguns dos benefícios que os estudantes reconheceram na atuação em projetos de voluntariado. Todo o impacto produzido pelo trabalho voluntário se associa ao desenvolvimento da visão humanitária necessária a uma boa prática médica. Além disso, os benefícios para o bem-estar social e psicológico em indivíduos que realizam o voluntariado já foram descritos em outros estudos 2,11 .

No Estudo 1, a falta de conhecimento sobre projetos de trabalho voluntário foi a segunda opção mais votada entre aqueles que não possuem interesse na atividade, e a falta de motivação foi a terceira opção mais votada. Este resultado expressa a importância de as universidades investirem em projetos de voluntariado e em sua divulgação, principalmente para contribuir na formação humanística médica. O Estudo 2 demonstra como o trabalho voluntário é um meio de os estudantes adquirirem mais conhecimento técnico-científico, treinarem suas habilidades interpessoais e desenvolverem uma visão humana do paciente. Desse modo, os Estudos 1 e 2 mostraram que o trabalho voluntário tem alcançado notoriedade na vida dos estudantes de Medicina da UFMG e que o não engajamento em tais projetos ocorre mais pela falta de conhecimento e divulgação dos mesmos do que pela falta de interesse dos alunos.

Algumas considerações devem ser feitas. O Estudo 1 teve divulgação e preenchimento do questionário pela internet. Não houve equilíbrio entre os participantes do Estudo 1 e o período em que se encontravam no curso, tendo havido maior concentração de estudantes que responderam ao questionário do oitavo e nono períodos.

Já no Estudo 2, participou um número pequeno de acadêmicos, aqueles que foram ao projeto "Cuidando da sua saúde em Ponto dos Volantes" (19 no total e 15 responderam ao questionário).

O voluntariado como uma atividade fomentada pelas universidades é um modo de introduzir o conhecimento sobre as condições socioeconômicas e as dificuldades de acesso à saúde de parte da população brasileira. Este conhecimento é importante para a conscientização sobre a necessidade de distribuição de profissionais pelo território nacional e para o comprometimento em ações de melhoria da saúde da população.

Considerando a relevância que o trabalho voluntário pode desempenhar na vida pessoal e profissional dos acadêmicos e os desafios que uma formação mais humana e com alto conteúdo técnico pode exigir, investir no desenvolvimento de projetos como o apresentado no Estudo 2 e outras atividades voluntárias é um modo de incorporar todos os benefícios à formação médica. A ampliação em nível nacional e de forma obrigatória de projetos de ensino de Internato Rural, em que os alunos possam ficar durante um trimestre letivo numa área de zona rural para prestar atendimentos, com auxílio da prefeitura local, é um exemplo. A instituição de intercâmbios de férias para localidades rurais, com parceria entre universidades, estados e prefeituras municipais, é outra forma de tornar as universidades mais ativas no processo de desenvolvimento do voluntariado. A realização de atendimentos em horários extras em comunidades próximas da universidade, com visitas domiciliares e mutirões, é outro exemplo, em menor escala, da prática voluntária médica.

As propostas apresentadas, principalmente as que envolvem os acadêmicos com a comunidade por um período de tempo mais prolongado, são alternativas plausíveis de envolvimento com o voluntariado. As parcerias que podem ser feitas auxiliam nos custos e na organização quanto ao gerenciamento dos alunos e ações desempenhadas por eles. Para que todas estas propostas sejam bem-sucedidas, é importante também considerar a divulgação de projetos sociais e trabalhar com a motivação dos estudantes, com a valorização e reflexão sobre o voluntariado.

As respostas dos acadêmicos de Medicina que participaram do projeto "Cuidando da sua saúde em Ponto dos Volantes" demonstram o impacto que o voluntariado teve em suas vidas e o potencial deste impacto para se refletir em mudanças futuras, principalmente no relacionamento com o paciente.

A percepção de uma atuação mais solidária e de um atendimento mais cuidadoso de acordo com as necessidades de cada paciente, tendo em vista as carências socioeconômicas envolvidas, é um aspecto do trabalho que contribuiu para a formação pessoal e acadêmica dos alunos, como pôde ser observado nas respostas fornecidas.

Apesar das limitações, é possível perceber as consequências positivas que o trabalho voluntário tem na vida dos estudantes de Medicina da UFMG. O projeto "Cuidando da sua saúde em Ponto dos Volantes" foi uma forma de fomentar o voluntariado e estimular o investimento das universidades brasileiras em projetos semelhantes para o envolvimento dos alunos. A divulgação do trabalho voluntário e de seus benefícios é uma forma imprescindível de expor e motivar os estudantes na atuação em projetos voltados à contribuição social. 


\section{REFERÊNCIAS}

1. International Labour Organization. ILO Manual on the measurement of volunteer work ILO Manual on the measurement of volunteer work. 1ํㅗ ed. Geneva; 2011

2. Ramos R, Brauchli R, Bauer G, Wehner T, Hämmig O. Busy Yet Socially Engaged: Volunteering, Work-Life Balance, and Health in the Working Population. J Occup Environ Med. 2015;57(2):164-72.

3. Piliavin JA, Siegl E. Health benefits of volunteering in the Wisconsin longitudinal study. J Health Soc Behav. 2007;48(4): 450-464.

4. IBGE. Brasil: IBGE - Instituto Brasileiro de Geografia e Estatística; 2017 [citado 2018 março 26]. Notícias. Disponível em: https://agenciadenoticias.ibge.gov.br/agencia-noticias/2012-agencia-de-noticias / noticias / 20618-cerca-de-6-5-milhoes-de-pessoas-fazem-trabalho-voluntario-no-pais.html

5. Bureau of Labor Statistics [internet]. EUA: Bureau of Labor Statistics; 2015 [citado 2016 fev 25]. Economic Releases. Disponível em: https://www.bls.gov/news.release/ volun.nr0.htm

6. Clary EG, Snyder M, Ridge RD, Copeland J, Stukas AA, Haugen J, et al. Understanding and Assessing the Motivations of Volunteers: A Functional Approach. J Pers Soc Psychol. 1998;74(6):1516-30.

7. Blasco GP. A Arte Médica (I): a formação e as virtudes do médico. RBM Especial Oncologia. 2013;69:9-17.

8. IBGE [internet]. Brasil: IBGE - Instituto Brasileiro de Geografia e Estatística; 2018. Projeção da população. Disponível em: https://www.ibge.gov.br/apps/populacao/ projecao//index.html

9. Scheffer M, Cassenote A, Guilloux AGA, Miotto BA, Mainardi GM. Demografia Médica no Brasil 2018. São Paulo: Departamento de Medicina Preventiva da Faculdade de Medicina da USP; Conselho Regional de Medicina do Estado de São Paulo; Conselho Federal de Medicina, 2018.
10. IBGE [internet]. Brasil: IBGE - Instituto Brasileiro de Geografia e Estatística; 2017. Panorama. Disponível em: https:/ / cidades.ibge.gov.br/brasil/mg/ponto-dos-volantes/panorama

11. Willigen VM. Differential Benefits of Volunteering Across the Life Course. J Gerontol. 2000;55B (5): 308-18.

\section{CONTRIBUIÇÃO DOS AUTORES}

Fernanda Mendes Amorim foi responsável pela concepção e desenho deste estudo, da análise e interpretação dos dados assim como da redação e revisão crítica para conteúdo intelectual. Luís Felipe José Ravic de Miranda contribuiu para a concepção e desenho do estudo e revisão crítica para conteúdo intelectual significativo. Ana Paula Bernardes Real contribuiu para a aquisição de dados, revisão das referências e elaboração de versão inicial do artigo e aprovação final. Gustavo Antunes Rodrigues Duarte contribui para análise e interpretação de dados, elaboração de versão inicial do artigo e aprovação final. Juliana Toledo Mesquita contribuiu para a concepção e desenho do estudo, análise e interpretação de dados, revisão crítica para conteúdo intelectual. Bruno Cézar Lage Cota contribuiu para a análise dos dados e revisão crítica para conteúdo intelectual.

\section{CONFLITO DE INTERESSES}

Não houve conflito de interesses para a realização deste estudo.

\section{ENDEREÇO PARA CORRESPONDÊNCIA}

Avenida Bernardo Monteiro, 1338, Funcionários.

Belo Horizonte CEP: 30140-285 MG

E-mail: ravicmiranda@gmail.com

Corresponding author - Prof. Dr. Luís Felipe José Ravic de Miranda,

Prof. Adjunto - Departamento de Clínica Médica

Faculdade de Medicina - UFMG 